\title{
Breaking the permeability-selectivity trade- off in thin-film composite polyamide membranes with a PEG-b-PSF-b-PEG block copolymer ultrafiltration membrane support through post-annealing treatment
}

\author{
Xiaochan An $\mathbb{D}^{1,2,3}$, Yunxia Hu', Ning Wang ${ }^{2}$, Tao Wang ${ }^{2}$ and Zhongyun Liu ${ }^{2}$
}

\begin{abstract}
In this work, amphiphilic polyethylene glycol-block-polysulfone-block-polyethylene glycol (PEG-b-PSF-b-PEG) was used as a membrane support to fabricate a high-performance thin-film composite (TFC) forward osmosis (FO) membrane. A facile post-annealing approach was developed to simultaneously improve the water permeability and antifouling property of the TFC membrane having the PEG-b-PSF-b-PEG support without sacrificing its selectivity. The experimental results illustrate that a highly crosslinked polyamide with low reverse salt flux could be formed on the PEG-b-PSF-b-PEG support, and the post-annealing treatment could tailor the membrane structure and properties of the PEG-b-PSF-b-PEG support to decrease its structure parameter without affecting the polyamide. The annealed TFC membrane exhibited excellent permeability-selectivity, with a high $A / B$ ratio of 19.6 bar $^{-1}$ (water permeability coefficient $A$ of $1.76 \mathrm{LMH}_{\mathrm{bar}}{ }^{-1}$ and $\mathrm{NaCl}$ permeability coefficient $B$ of $0.09 \mathrm{LMH}$ ). The static and dynamic antifouling performances of the annealed TFC membrane were also demonstrated, finding that little bovine serum albumin (BSA) was adsorbed on the PEG-b-PSF-b-PEG support surface, and a reduced water flux decline and a high water recovery were achieved compared with those of the control sample. Our work demonstrates that the amphiphilic PEG-b-PSF-bPEG membrane can work as an ideal TFC support to break the permeability-selectivity trade-off of the TFC FO membrane and to improve its antifouling properties through post-annealing treatment.
\end{abstract}

\section{Introduction}

Freshwater shortage and the clean energy crisis are becoming the most important global challenges with increasing population growth, accelerating urbanization,

\footnotetext{
Correspondence: Yunxia Hu (yunxiahu@yic.ac.cn)

'State Key Laboratory of Separation Membranes and Membrane Processes, National Center for International Research on Membrane Science and Technology, School of Materials Science and Engineering, Tianjin Polytechnic University, Tianjin 300387, PR China

${ }^{2}$ CAS Key Laboratory of Coastal Environmental Processes and Ecological Remediation, Research Center for Coastal Environmental Engineering and Technology of Shandong Province, Yantai Institute of Coastal Zone Research, Chinese Academy of Sciences, Yantai, Shandong Province 264003, PR China Full list of author information is available at the end of the article.
}

and worsening climate change ${ }^{1,2}$. Advanced technologies are urgently needed to economically produce eco-friendly freshwater and clean energy. Recently, forward osmosis (FO) has been developed as an advanced membrane technology for desalination, wastewater reclamation, power generation, etc. FO has been extensively investigated as an easy-operation and low-fouling membrane process to introduce spontaneous water transport under an osmotic-pressure driving force across a semipermeable membrane ${ }^{3,4}$. High-performance semipermeable membranes play a key role in the FO process, and thin-filmcomposite (TFC) polyamide membranes are the state-ofthe-art choice for current FO membranes having great 
water permeability and salt rejection ${ }^{2,5}$. TFC polyamide membranes possess a highly crosslinked polyamide active layer formed through interfacial polymerization on top of a polymeric ultrafiltration (UF) membrane support ${ }^{5}$. Different from other reverse osmosis and nanofiltration membranes, not only the polyamide active layer but also the microporous support determine the water permeability of FO membranes due to the internal concentration polarization (ICP) effect of the support ${ }^{6,7}$. The water flux and salt rejection of TFC FO membranes have great potential to be further improved through optimization of the structure and properties of the porous support $^{8}$.

Previous studies have identified that the ideal ICPsuppressed support of a TFC FO membrane should have minimal thickness and tortuosity and a high porosity to provide a short path for drawing solute, and its material chemistry should have suitable intrinsic hydrophilicity, mechanical strength, and chemical stability ${ }^{9,10}$. Thus, many efforts have been devoted to fabricating such a support layer to reduce ICP in TFC FO membranes, including optimizing the phase inversion process to make a finger-like pore structure with large surface pores ${ }^{5,11}$, blending nanomaterials into a polymeric casting solution to improve the highly porous structure with a high surface hydrophilicity, and modifying the support layer with hydrophilic materials to enhance water transport ${ }^{12-14}$. The water flux of fabricated TFC membranes is thereby increased through the use of such modified supports. However, the fabricated TFC membranes significantly lose their salt rejection and selectivity ${ }^{9,15}$. This drawback occurs because the large surface pores and hydrophilic surfaces of the support are not beneficial to forming a highly crosslinked polyamide with great salt rejection through the fast interfacial polymerization of two highly reactive molecules, $\mathrm{m}$-phenylenediamine (MPD) and trimesoyl chloride (TMC) ${ }^{16,17}$. Thus, enormous challenges to fabricating high-performance TFC FO membranes with a high water flux and great salt rejection through the formation of a highly permeable and selective polyamide layer on the ICP-suppressed porous support persist ${ }^{16,18}$.

Therefore, it is highly desirable to fabricate an ideal support that has a favorable surface to form the permeable and selective polyamide layer through interfacial polymerization and to tailor the membrane structure and properties to minimize the structure parameter $S$ and reduce ICP. An amphiphilic polysulfone-based block copolymer (polyethylene glycol-b-polysulfone-bpolyethylene glycol, PEG-b-PSF-b-PEG) may contain great potential in the fabrication of such an ideal TFC support. Our previous work has found that this polysulfone-based block copolymer could be used to cast a UF membrane having similar surface properties as those of the polysulfone (PSF) UF membrane. Such a material may provide a favorable support surface for the formation of highly crosslinked polyamide with great salt rejection ${ }^{19}$, as the PSF UF membrane has been well demonstrated as a TFC support for the formation of highly selective polyamide with more than $98 \% \mathrm{NaCl}$ rejection ${ }^{11,20}$. Importantly, the surface properties and membrane structure of the block copolymer UF membrane could be finely tailored by the self-assembly of block copolymers through the post-annealing process ${ }^{21}$. Consequently, the membrane surface hydrophilicity and water permeability of the annealed block copolymer UF membrane were greatly improved ${ }^{19}$, indicating a significant decrease in the water transport resistance of the block copolymer UF membrane with a low structure parameter $S$ due to the annealing. However, to date, no work has been performed to cast this polysulfone-based block copolymer UF membrane as a support to fabricate a high-performance TFC FO membrane.

Herein, we are the first to use this amphiphilic polysulfone-based block copolymer (PEG-b-PSF-b-PEG) to cast a support for the fabrication of a high-performance TFC FO membrane. We prepared the block copolymer membrane support via the nonsolvent-induced phase separation (NIPS) method and utilized the interfacial polymerization of MPD and TMC to form the polyamide selective layer on top of the block copolymer membrane support. An annealing treatment was performed to tailor the structure and properties of the polysulfone-based block copolymer membrane support and the TFC polyamide membrane. The surface morphology and structural and transport properties of both the block copolymer membrane support and the formed polyamide were characterized before and after the annealing treatment. Then, the impacts of the annealing treatment on the block copolymer membrane support and the formation of polyamide were discussed. Finally, bovine serum albumin (BSA) was used as a protein foulant model to investigate the static and dynamic BSA fouling performance of the TFC FO membrane, with the block copolymer membrane support facing the protein-containing feed solution. We thus provide critical insights on selecting block copolymer membrane materials as the TFC membrane support and investigating the impact of the block copolymer membrane support on the formation of polyamide.

\section{Materials and methods \\ Materials and chemicals}

Polysulfone Udel P-3500 (PSF, $M_{n}$ : 22,000 Da) was purchased from Solvay Advanced Polymers (Alpharetta, GA, USA). The polysulfone-based block copolymer (polyethylene glycol-b-polysulfone-block-polyethylene glycol, simplified as PEG-b-PSF-b-PEG, $M_{n}: 16,000 \mathrm{Da}$, PDI: 2.05, PEG: $35.5 \mathrm{wt} \%)$ was synthesized and characterized following our previous report $^{19}$. 1,3-Phenylenediamine 
(MPD, >99\%), 1,3,5-benzenetricarbonyl trichloride (TMC, 98\%), and N-methyl-2-pyrrolidone (NMP, 99.5\%) were obtained from Sigma-Aldrich (USA). N-hexane and sodium chloride $(\mathrm{NaCl})$ were purchased from Sinopharm Chemical Reagent (China). All chemicals were used as received.

\section{Fabrication of TFC FO membranes}

The TFC FO membranes were fabricated through two steps following the reported protocol ${ }^{5}$, which includes the preparation of the porous polymeric support via the NIPS method and the fabrication of the polyamide selective layer via interfacial polymerization. First, $12 \mathrm{wt} \%$ homogenous casting solutions of PSF or PEG-b-PSF-b-PEG were prepared in NMP and then degassed overnight. A steel casting knife (Tianjin HongJuLi Experimental Equipment Factory, China) with a $150 \mu \mathrm{m}$ gate height was used to cast the PSF or PEG-b-PSF-b-PEG solution onto the PET nonwoven fabric prewetted with NMP. Upon immediate immersion in a water coagulation bath for $10 \mathrm{~min}$ at room temperature, a white PSF or PEG-b-PSFb-PEG membrane formed and was transferred to deionized (DI) water for storage. All the resulting support membranes were stored in DI water before interfacial polymerization. To form the polyamide active layer on the support membrane (including the PSF, pristine block copolymer, and annealed block copolymer supports), the top selective surface of the wet support membrane was immersed in an MPD aqueous solution (3.4wt\%) for 2 min. After removing the excess MPD with a rubber roller, the MPD-containing support was soaked in a TMC hexane solution $(0.15 \mathrm{wt} \%)$ for $60 \mathrm{~s}$ to form the polyamide active layer, followed by curing in DI water at $95^{\circ} \mathrm{C}$ for $120 \mathrm{~s}$. Three different types of TFC FO membranes were fabricated, as illustrated in Scheme 1. The TFC FO membrane with the PSF support was used as a control sample.

\section{Post-annealing treatment of membranes}

The prepared membranes were heated in a water bath at $90^{\circ} \mathrm{C}$ for $16 \mathrm{~h}$ following our reported procedure ${ }^{19}$; the membranes were PSF or block copolymer membrane supports or TFC polyamide membranes with PSF or block copolymer supports. All membranes were stored in $4{ }^{\circ} \mathrm{C}$ DI water before use or characterization.

\section{Characterization of TFC FO membrane}

The surface and cross-sectional morphologies of the polymeric support and TFC FO membranes were observed by scanning electron microscopy (SEM, Hitachi S-4800, Japan). Liquid nitrogen was used to crack the membranes to observe the cross-sectional structure. Before observation, all samples were dried in a vacuum oven $\left(40^{\circ} \mathrm{C}, 12 \mathrm{~h}\right)$ and coated with platinum (Pt) $(120 \mathrm{~s})$ using an EMITECH SC7620 sputtering machine. The software Nano Measure was employed to measure the pore size of the support surface and the thickness of the dense skin layer. Each datum was reported as the average value of three parallel specimens.

The water contact angle of membrane surfaces was measured using a Data Physics optical instrument (OCA20, Germany) equipped with the Drop Shape Analysis software (SCA20 Version 2). Upon dispensing $2 \mu \mathrm{L}$ of a DI water droplet on the membrane surface for $60 \mathrm{~s}$, the shape of the water droplet was recorded, and the contact angle was thus calculated. Each datum was an average value of three individual parallel samples.

The roughness of the polyamide surface of the TFC FO membranes was measured by atomic force microscopy (AFM, Veeco/Digital Instruments, Santa Barbara, CA). NanoScope software version 8.10 and NanoScope Analysis software version 1.10 were used for image collection and data analysis.

X-ray photoelectron spectroscopy (XPS, Thermo EscaLab 250Xi, USA) with a monochromatic Al $\mathrm{K} \alpha$ source from Thermo Fisher Scientific was used to examine the elemental compositions of the polymeric support surface and the polyamide surface of the TFC membranes. The photoelectron take-off angle was $60^{\circ}$, and the full range of data was collected by a survey scan from 200 to $1200 \mathrm{eV}$ with a step size of $1 \mathrm{eV}$. The crosslinking degree of the polyamide selective layer was calculated according to the $\mathrm{O} / \mathrm{N}$ element ratio ${ }^{16}$. The $\mathrm{O} / \mathrm{N}$ ratio of the fully crosslinked polyamide $\left[\mathrm{C}_{15} \mathrm{H}_{10} \mathrm{O}_{3} \mathrm{~N}_{3}\right]$ is 1 , while that of the fully linear structure of $\left[\mathrm{C}_{15} \mathrm{H}_{10} \mathrm{O}_{4} \mathrm{~N}_{2}\right]$ is 2 . The relative fractions of the crosslinked portion, $m$, and the linear portion, $n$, were calculated following the reported equations (1) and $(2)^{22}$.

$$
\begin{aligned}
& m+n=1 \\
& \frac{O}{N}=\frac{3 m+4 n}{3 m+2 n}
\end{aligned}
$$

\section{Transport property measurements of TFC FO membrane}

The water flux and the salt reverse flux of the TFC FO membranes were measured using a laboratory-scale cross-flow FO system equipped with a flat-sheet membrane cell with a $38.52 \mathrm{~cm}^{2}$ effective membrane area ${ }^{23}$. DI water $(2 \mathrm{~L})$ and a $2 \mathrm{~L} \mathrm{NaCl}$ solution $(0.5-2.0 \mathrm{M})$ were used as the feed and draw solutions, respectively. The FO test ran at $25 \pm 1{ }^{\circ} \mathrm{C}$ with a $0.5 \mathrm{~L} / \mathrm{min}$ cross-flow rate for both feed and draw solutions. The membranes were evaluated under both AL-DS (active layer facing the draw solution) and AL-FS (active layer facing the feed solution) orientations. The water flux was measured as the water weight gain of the draw side using a digital balance (ME3002, Mettler-Toledo, Switzerland), and the reverse salt flux was 


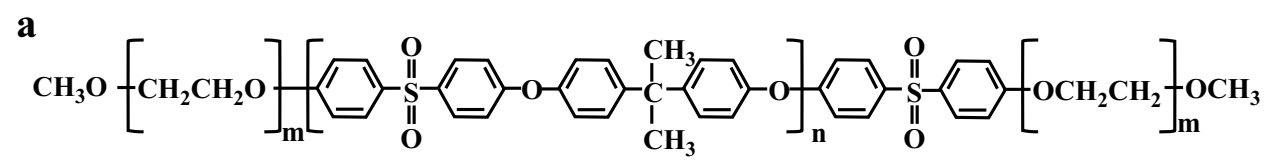

b

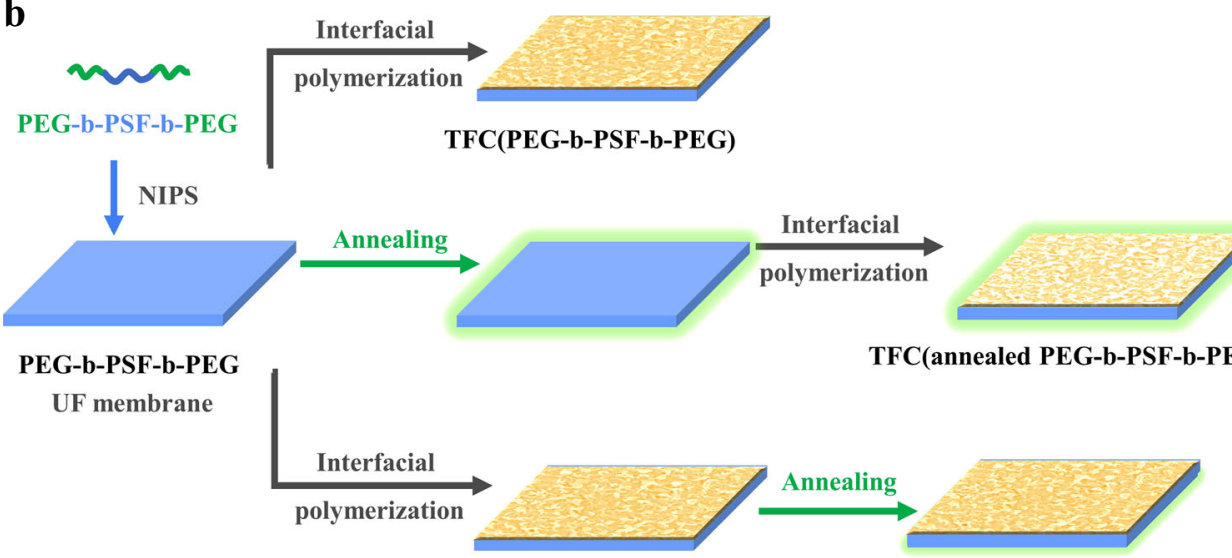

Annealed TFC(PEG-b-PSF-b-PEG)

Scheme 1 Molecular structure of the PEG-b-PSF-b-PEG block copolymer (a) and the fabrication of TFC FO membranes using the PEG-b-PSF-b-PEG UF membrane support with different treatments, including $\mathbf{b}$ pre-annealing and post-annealing. Interfacial polymerization: the samples were immersed in the MPD aqueous solution (3.4 wt \%) for $2 \mathrm{~min}$ and the TMC hexane solution $(0.15 \mathrm{wt} \%)$ for $60 \mathrm{~s}$ and then cured in a water bath at $95^{\circ} \mathrm{C}$ for $120 \mathrm{~s}$. Annealing treatment: the samples were heated in a water bath at $90^{\circ} \mathrm{C}$ for $16 \mathrm{~h}$

measured as the salt weight gain of the feed side using a conductivity meter (CON2700, Eutech, USA).

The water permeability coefficient $(A)$, salt permeability coefficient $(B)$, and structural parameter $(S)$ of different TFC FO membranes were determined in the abovementioned FO system according to the reported proto$\mathrm{Col}^{24}$. The detailed experimental procedures are given in the Supporting Information.

\section{Static and dynamic antifouling evaluations of the TFC FO membranes}

BSA was chosen as a representative protein foulant to investigate the antifouling performance of the control TFC(PSF) and annealed TFC(PEG-b-PSF-b-PEG) membranes. An Olympus confocal laser scanning microscope (CLSM, Fluo View FV1000, Japan) was used to observe the adsorption of fluorescein isothiocyanate (FITC)labeled BSA, which was prepared according to the reported protocol ${ }^{25}$, on the support layers of the TFC membranes. The TFC(PSF) and annealed TFC(PEG-bPSF-b-PEG) membranes were soaked and incubated in $0.5 \mathrm{mg} \mathrm{mL}^{-1}$ FITC-labeled BSA/PBS solution for $2 \mathrm{~h}$. Subsequently, all the membranes were gently rinsed thrice with PBS solution and DI water. Then, fluorescent photographs of the samples were taken with an excitation filter of $488 \mathrm{~nm}$ and an emission filter of $519 \mathrm{~nm}$ using the CLSM.
Following our previous study, the dynamic fouling experiments were carried out in the abovementioned crossflow FO setup with BSA as the model protein foulant ${ }^{26}$. The annealed TFC(PEG-b-PSF-b-PEG) and control TFC(PSF) membranes were oriented in the AL-DS mode. Simulated river water $(0.4 \mathrm{mM} \mathrm{NaCl}, 0.2 \mathrm{mM} \mathrm{NaHCO}$, $0.3 \mathrm{mM} \mathrm{CaCl}_{2}$, and $150 \mathrm{mg} / \mathrm{L} \mathrm{BSA}$ ) was used as a feed solution, and the concentration of the $\mathrm{NaCl}$ draw solution was tailored to obtain an initial water flux of the TFC FO membrane of approximately $20 \mathrm{LMH}$. Quantitative analysis of the foulants was performed by measuring the total protein extracted from the membrane surface using the reported BCA method ${ }^{27}$.

\section{Results and discussion \\ Post-annealing treatment improves the filtration performances and surface properties of the block copolymer UF membrane support}

In our previous work $^{19}$, the amphiphilic polysulfonebased block copolymer was used to fabricate a porous UF membrane with an asymmetric structure having an ultrathin sponge-like skin layer on top of a finger-like pore matrix through the NIPS method. This membrane exhibited very similar surface properties to those of the traditional PSF UF membrane being widely used as the TFC polyamide membrane support. Importantly, the membrane surface properties, including the surface 


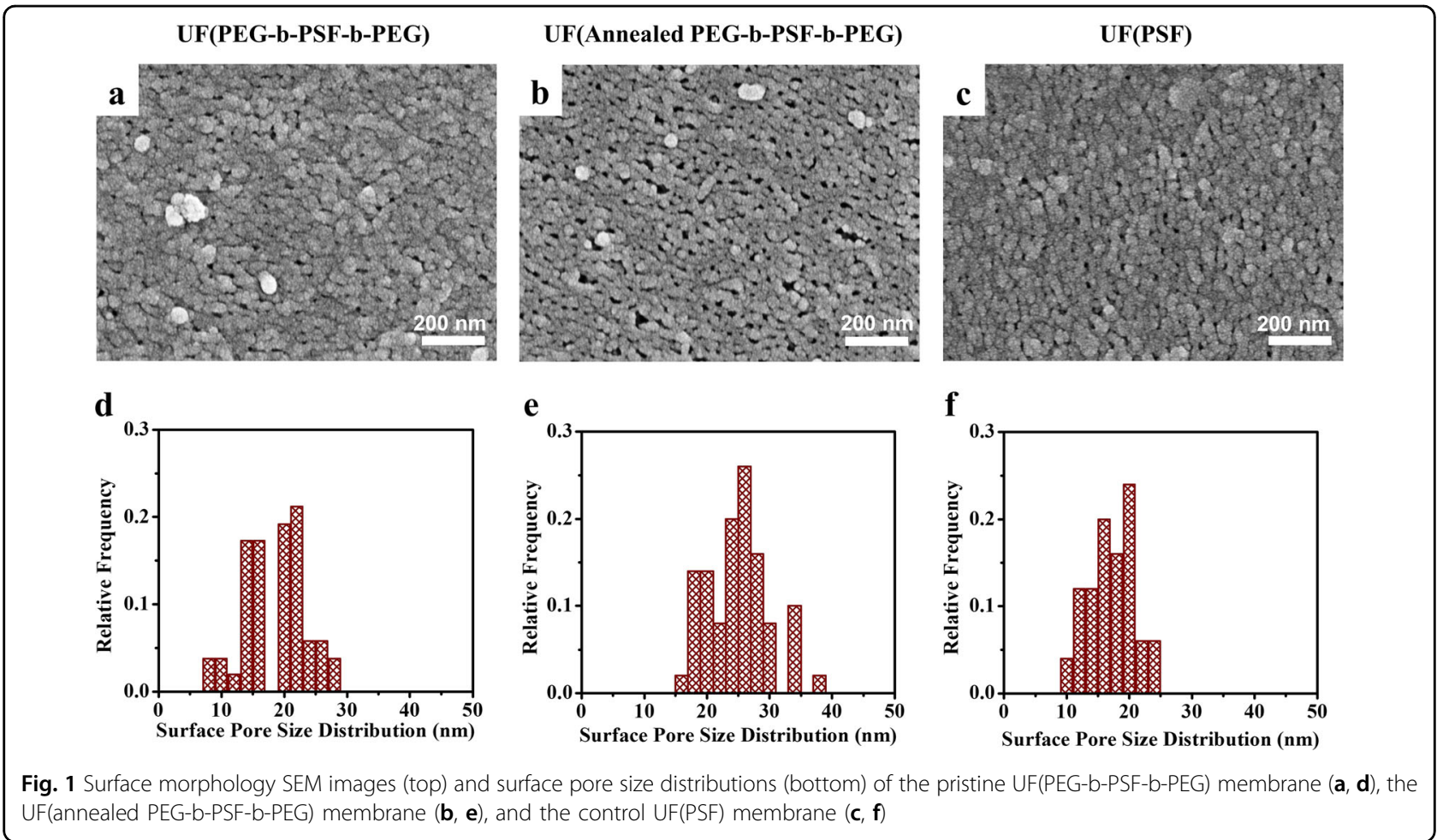

hydrophilicity, surface pore size, surface porosity, and surface roughness, could be finely tailored through the directed self-assembly of PSF blocks and PEG blocks via solvothermal annealing treatment in hot water $\left(90^{\circ} \mathrm{C}\right.$, $16 \mathrm{~h})^{19}$. In this work, a $150 \mu \mathrm{m}$ thick block copolymer UF membrane was casted on nonwoven PET fabric as a backing support with potentially reduced ICP effect and strengthened mechanical strength. The mean surface pore size of the block copolymer membrane casted on the PET fabric obviously increased from $20 \pm 1$ to $26 \pm 3 \mathrm{~nm}$ upon the post-annealing treatment, as shown in Fig. 1 and Table 1; these values were significantly larger than the corresponding values of 12 and $16 \mathrm{~nm}$ for the block copolymer membrane casted on the glass before and after the annealing treatment ${ }^{19}$. This result illustrates that the nonwoven PET backing support can prevent the surface pore shrinkage of the block copolymer membrane, a reported phenomenon ${ }^{28}$. In addition, the block copolymer membrane surface porosity was measured based on the SEM images of membrane surface morphologies shown in Fig. 1, and the pristine block copolymer UF membrane has a surface porosity of $7.5 \pm 1.2 \%$, very close to the value of $6.8 \pm 1.4 \%$ for the PSF control membrane. The annealing process dramatically increased the surface porosity of the annealed block copolymer membrane to $14.4 \pm 3.3 \%$, as well as its membrane porosity to $80.4 \pm$ 2.7\%; these values are substantially higher than those of the reported polymeric UF membranes fabricated through the phase inversion method ${ }^{29-31}$. As a control, there was no apparent change in the PSF UF membrane upon annealing treatment.

The membrane filtration properties were measured, finding that the pure water flux of the annealed block copolymer UF membrane was $1582 \pm 63 \mathrm{LMH} /$ bar, significantly higher than the value of $1231 \mathrm{LMH} /$ bar reported in our previous work ${ }^{19}$ owing to the decreased mass transport resistance from the thinner membrane thickness and larger pore size. Furthermore, the annealed block copolymer UF membrane exhibited an increased water flux compared with the pristine block copolymer membrane and PSF control membrane, benefiting from the augmented pore size and porosity along with the obvious decrease in the skin layer thickness, as shown in Table 1. More importantly, the annealed block copolymer membrane also presented a dramatically decreased water contact angle of $12.1 \pm 5.1^{\circ}$ compared with the values of $78.4 \pm 2.7^{\circ}$ for the pristine block copolymer UF membrane and $85.1 \pm 3.2^{\circ}$ for the pristine PSF membrane ${ }^{32}$. This observation further confirms the improved surface hydrophilicity from the PEG surface enrichment upon the directed self-assembly of the polysulfone-based block copolymer via the post-annealing treatment (Table 1 and Figure S2). Additionally, the great advantage of this amphiphilic polysulfone-based block copolymer was in casting a membrane having a strong mechanical strength $(5.56 \pm 0.53 \mathrm{MPa}$, very close to that of the polysulfone 
Table 1 Characteristic properties of the UF(PEG-b-PSF-b-PEG) membrane, the UF(annealed PEG-b-PSF-b-PEG) membrane, and the controlled UF(PSF) membrane

\begin{tabular}{lccc}
\hline Samples & UF(PEG-b-PSF-b-PEG) & UF(annealed PEG-b-PSF-b-PEG) & UF(PSF) \\
\hline Porosity (\%) & $74.0 \pm 4.1$ & $80.4 \pm 2.7$ & $73.7 \pm 3.3$ \\
Surface porosity (\%) & $7.5 \pm 1.2$ & $14.4 \pm 3.3$ & $6.8 \pm 1.4$ \\
Skin layer thickness (nm) & $288 \pm 12$ & $259 \pm 18$ & $280 \pm 10$ \\
Pure water flux ${ }^{\mathrm{a}}$ (LMH/bar) & $982 \pm 140$ & $1582 \pm 63$ & $855 \pm 219$ \\
Salt diffusion coefficient ${ }^{\mathrm{b}}\left(\mathrm{mmol} \mathrm{L}^{-1} \mathrm{~min}^{-1}\right.$ ) & $0.50 \pm 0.10$ & $0.62 \pm 0.17$ & $0.28 \pm 0.08$ \\
Water contact angle (degree) & $78.4 \pm 2.7$ & $12.1 \pm 5.1$ & $85.1 \pm 3.2$ \\
Mean surface pore size (nm) & $20 \pm 1$ & $26 \pm 3$ & $18 \pm 2$ \\
\hline
\end{tabular}

${ }^{\text {a }}$ Pure water flux of all UF membranes was determined using the dead-end cell with DI water at $25^{\circ} \mathrm{C}$ and under 1 bar of the hydraulic pressure

${ }^{b}$ Salt diffusion coefficient $\left(k, \mathrm{mmol} \mathrm{L}^{-1} \mathrm{~min}^{-1}\right)$ : the diffusion rate of $\mathrm{NaCl}$ solution across the membrane $\left(c, \mathrm{mmol} \mathrm{L}^{-1}\right)$ per time $(t, \mathrm{~min})$

membrane) compared with that of other polystyrenebased block copolymers $(2-3 \mathrm{MPa})$ for the UF membrane fabrication $^{33,34}$.

The salt diffusion coefficient of the block copolymer membrane was measured to illustrate its performance in accelerating salt diffusion through the membrane and thus investigate its potential in reducing the ICP of the TFC FO membrane when used as the support. As shown in Table 1, the annealed block copolymer UF membrane presented a distinctive increase of its salt diffusion coefficient by $24 \%$ to $0.62 \mathrm{mmol} \mathrm{L}^{-1} \mathrm{~min}^{-1}$, in comparison with the value of $0.50 \mathrm{mmol} \mathrm{L}^{-1} \mathrm{~min}^{-1}$ for the pristine block copolymer membrane. In contrast, the salt diffusion coefficient of the PSF membrane was only $0.28 \mathrm{mmol} \mathrm{L}^{-1}$ $\mathrm{min}^{-1}$ due to its low porosity and low water permeability. All these findings illustrate that the post-annealing treatment can finely tailor the surface pore size, surface porosity, and surface hydrophilicity to yield accelerated salt diffusion of the block copolymer membrane support, which has great potential for use as the support of TFC FO membranes with reduced ICP.

Surface properties of the block copolymer UF membrane support affect the formation of the polyamide layer via interfacial polymerization

The polyamide active layer was formed on the block copolymer membrane support to fabricate a TFC FO membrane through the interfacial polarization of MPD and TMC, and rich characteristic "lotus-like" features were observed on the polyamide surface, as shown in Fig. 2 and Figure S5, similar to the polyamide formed on the PSF membrane support. Interestingly, with the resulting small surface area of the polyamide formed on the annealed block copolymer membrane support, the lotus leaf size was obviously smaller and the lotus height shorter than those of the polyamide formed on the pristine block copolymer membrane support and the PSF membrane support. Moreover, the surface roughness $\left(R_{a}\right)$ of the polyamide formed on the annealed block copolymer membrane support was $61 \pm 15 \mathrm{~nm}$, substantially lower than the values of $86 \pm 7$ and $93 \pm 9 \mathrm{~nm}$ for the polyamide layers formed on the pristine block copolymer and PSF membrane supports, respectively. X-ray photoelectron spectrometry (XPS) was employed to further characterize the surface elemental compositions and thus the crosslinking degree of the polyamide layers formed on different membrane supports. The results shown in Table 2 reveal that the polyamide formed on the annealed block copolymer membrane support had a linear structure with a very low crosslinking degree of approximately 0.1 , significantly lower than the value of 0.5 for the polyamide layers formed on the pristine block copolymer and PSF membrane supports. Therefore, it can be concluded that the surface properties of the annealed block copolymer membrane support significantly affected the formation of the polyamide, and its hydrophilic surface with large surface pore size was favorable to forming a linear polyamide structure with a very low crosslinking degree and a small surface area. The low crosslinking degree and low selectivity of the polyamide formed on the annealed PEG-b-PSF-b-PEG support can be explained by a conceptual model of the polyamide formation mechanism, as proposed by Hoek ${ }^{16}$. The physicochemical properties of the support affect the distribution of MPD in the support, the diffusion of MPD from the support surface to the organic phase and thus the reaction with TMC. The annealed PEG-b-PSF-b-PEG support is hydrophilic with the PEG surface enrichment, and the diffusion of MPD is slowed down because of the favorable interaction of MPD with the polar groups of the PEG block. Then, the reactivity of low concentration MPD with TMC decreases, which leads to the formation of polyamide with a low crosslinking degree and low selectivity ${ }^{22,35,36}$. Noticeably, the polyamide formed on the pristine block copolymer 


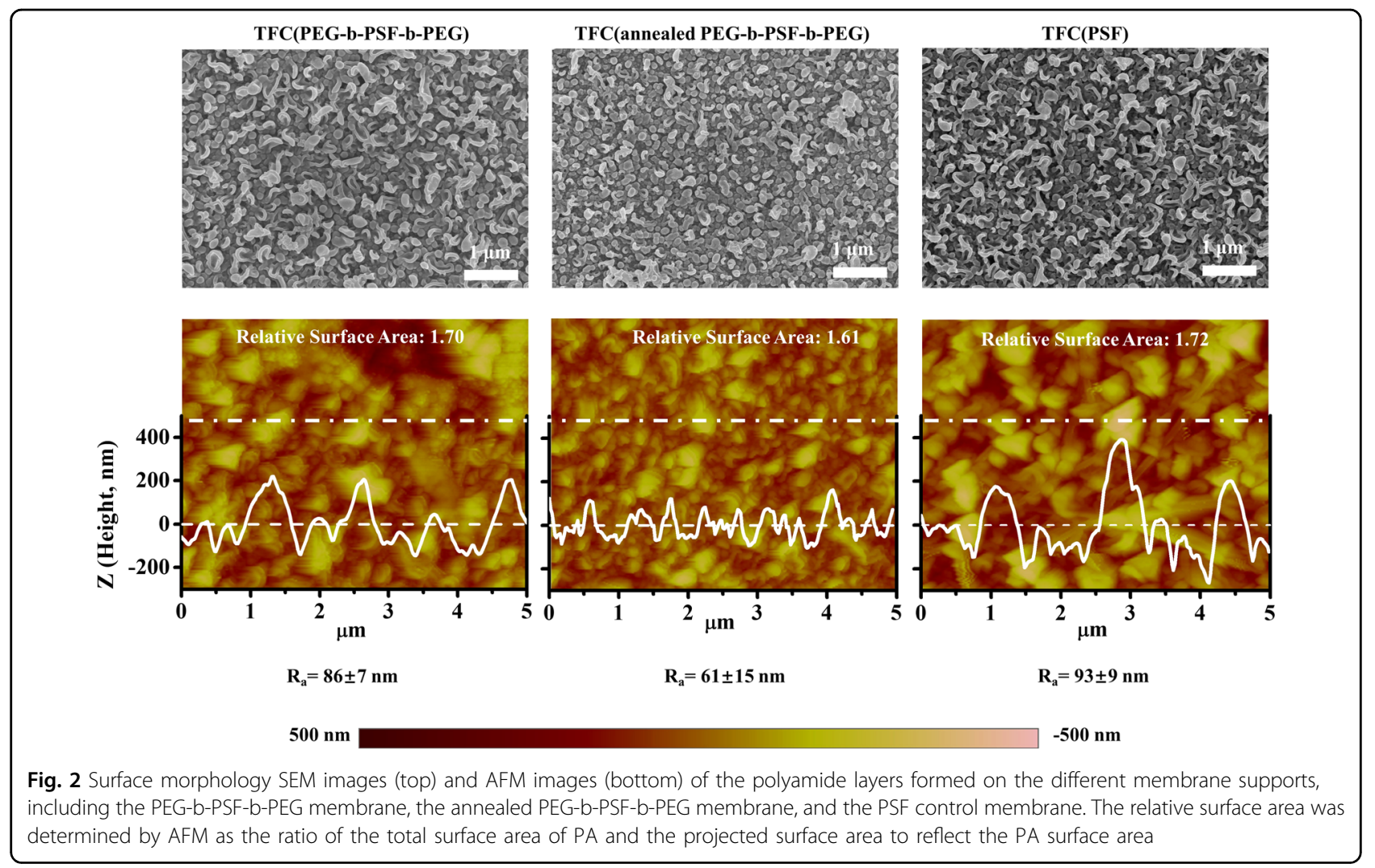

membrane support exhibited high crosslinking degree and surface roughness similar to that formed on the PSF membrane support owing to the similar surface properties of the pristine block copolymer and the PSF membrane supports, including the surface pore size, surface porosity, surface hydrophobicity, and surface roughness, as shown in Table 1.

The permeability and selectivity of the fabricated TFC polyamide membranes with different supports were measured to investigate their transport properties. Their water permeability coefficient $(A)$ and salt permeability coefficient $(B)$ were tested on a lab-scale FO cross-flow set-up. Figure $3 \mathrm{a}$ illustrates that the TFC polyamide membrane with the annealed block copolymer membrane support had an $A$ value of $1.22 \pm 0.04 \mathrm{LMH} / \mathrm{bar}$, close to the values of $1.11 \pm 0.21$ and $1.32 \pm 0.11 \mathrm{LMH} / \mathrm{bar}$ for the TFC polyamide membranes with the pristine block copolymer and PSF supports, respectively, indicating their similar water permeabilities. However, the TFC polyamide membrane with the annealed block copolymer support had a very high $B$ value of $0.29 \pm 0.05 \mathrm{LMH}$ owing to the very low crosslinking degree of the formed polyamide, a two-fold increase compared with the values of $0.13 \pm 0.04$ and $0.11 \pm 0.02 \mathrm{LMH}$ for the TFC polyamide membranes having the pristine block copolymer and PSF supports, correspondingly. Moreover, the reverse salt flux of the
TFC FO membranes were tested to further confirm that the polyamide formed on the annealed block copolymer membrane support had a very poor salt rejection of 7.8 $\mathrm{gMH}$ as its reverse salt flux (Fig. 3b), significantly higher than the values of 1.3 and $4.0 \mathrm{gMH}$ for those formed on the pristine block copolymer and PSF membrane supports.

Next, the structure parameter $S$ of the TFC polyamide membrane, which represents the water transport length across the support as a direct indicator of the ICP and reflects the effect of the TFC support on the performance of the FO membrane, was studied. As expected, the annealed block copolymer membrane support was found to decrease the structure parameter $S$ of the TFC membrane to $412 \pm 15 \mathrm{~nm}$ (Fig. 3a), compared with the values of $453 \pm 23$ and $421 \pm 34 \mathrm{~nm}$ obtained with the corresponding TFC supports of the pristine block copolymer membrane and the PSF membrane. The water flux is a key parameter of the FO membrane performance in real applications, representing the sum of contributions from $A, B$, and $S$. The TFC FO membranes with the pristine block copolymer membrane support and the PSF membrane support control presented similar high water fluxes of $\sim 30 \mathrm{LMH}$ and low reverse salt fluxes less than $4 \mathrm{gMH}$. With the annealed block copolymer support, the water flux of the TFC FO membrane slightly increased to 
Table 2 Relative atomic compositions (determined by Xray photoelectron spectroscopy, XPS) and the degree of cross-linking of polyamide formed on different membrane supports including the PEG-b-PSF-b-PEG membrane, the annealed PEG-b-PSF-b-PEG membrane, and the PSF control membrane

\begin{tabular}{|c|c|c|c|c|c|}
\hline \multirow[t]{2}{*}{ Samples } & \multicolumn{3}{|c|}{$\begin{array}{l}\text { Atomic } \\
\text { composition } \\
\text { from XPS (\%) }\end{array}$} & \multirow[t]{2}{*}{$\mathrm{O} / \mathrm{N}$} & \multirow[t]{2}{*}{$\begin{array}{l}\text { Degree of cross- } \\
\text { linking }\end{array}$} \\
\hline & $\mathrm{C}$ & 0 & $\mathrm{~N}$ & & \\
\hline $\begin{array}{l}\text { Fully cross-linked } \\
\text { (theoretically) }\end{array}$ & 75 & 12.5 & 12.5 & 1 & 1 \\
\hline Fully linear (theoretically) & 71.4 & 19.1 & 9.5 & 2 & 0 \\
\hline TFC(PEG-b-PSF-b-PEG) & 75.2 & 14.5 & 10.3 & 1.4 & 0.5 \\
\hline $\begin{array}{l}\text { TFC(annealed PEG-b-PSF- } \\
\text { b-PEG) }\end{array}$ & 69.0 & 20.3 & 10.7 & 1.9 & 0.1 \\
\hline TFC(PSF) & 73.1 & 15.8 & 11.2 & 1.4 & 0.5 \\
\hline
\end{tabular}

35.4 LMH owing to the reduced ICP from the smaller $S$, while its reverse salt flux was as high as $7.8 \mathrm{gMH}$ due to its high $B$ (Fig. 3b).

Therefore, all of these results illuminate that the annealed block copolymer membrane did not facilitate the formation of the highly selective polyamide as well as the pristine block copolymer membrane did but showed great potential in reducing the structure parameter $S$ and ICP of the TFC FO membrane.

\section{Post-annealing treatment breaks the} permeability-selectivity trade-off of the TFC FO membrane having the block copolymer UF membrane support

Learning from the aforementioned results, we first fabricated the TFC FO membrane by synthesizing a highly selective polyamide on the pristine block copolymer membrane support, followed by the post-annealing treatment to tailor the structure and properties of the block copolymer membrane support to reduce $S$ and ICP. To distinguish it from the FO membrane with the annealed block copolymer membrane support, labeled as the TFC(annealed PEG-b-PSF-b-PEG), we named this FO membrane the annealed TFC(PEG-b-PSF-b-PEG) since the TFC FO membrane with the block copolymer membrane support was fabricated first and then annealed. Interestingly, the post-annealing treatment significantly improved the water permeability coefficient $A$ of the annealed TFC(PEG-b-PSF-b-PEG) membrane to 1.76 $\mathrm{LMH} /$ bar (Fig. $4 \mathrm{a}$ ), an increase of $58 \%$ over that of the TFC membrane without annealing, but did not affect the membrane selectivity, having a very low salt permeability coefficient $B$ of $0.09 \mathrm{LMH}$, close to the values of 0.13 and
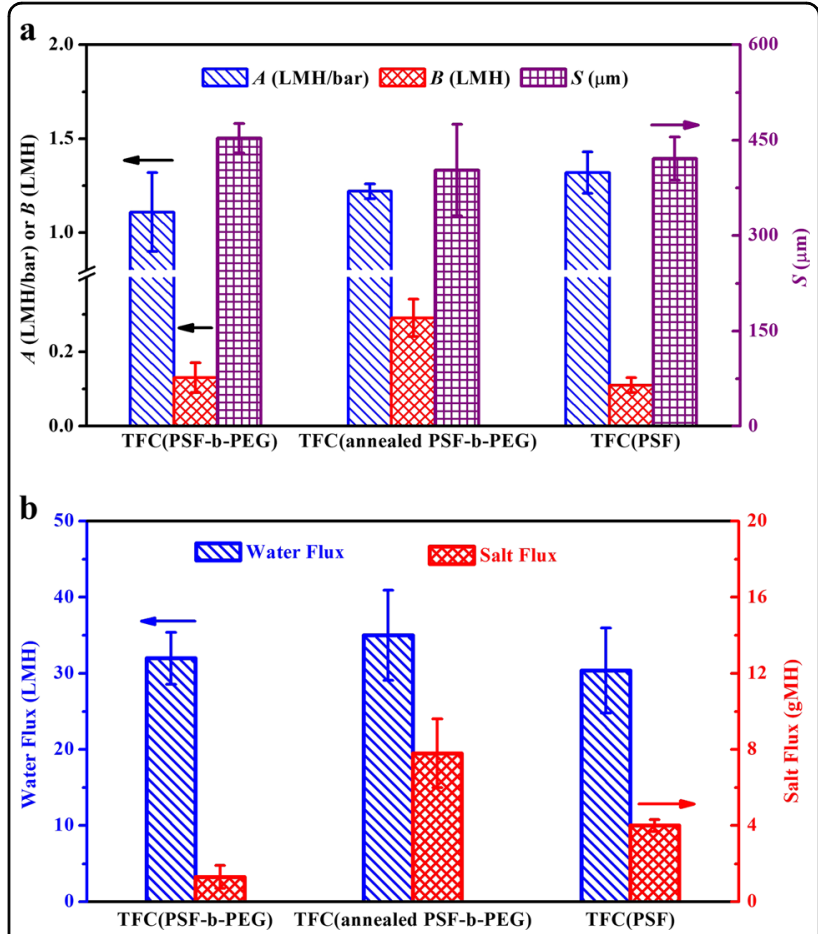

Fig. 3 The intrinsic transport parameters of various TFC FO membranes, including the water permeability coefficient $A$, salt permeability coefficient $B$, and structural parameter $S(\mathbf{a})$, and the water flux and reverse salt flux of the TFC FO membranes with different supports, including the PEG-b-PSF-b-PEG membrane, the annealed PEG-b-PSF-b-PEG membrane and the PSF membrane, as operated in the AL-DS mode with $1.0 \mathrm{M} \mathrm{NaCl}$ and DI water as draw and feed solutions, respectively (b)

0.11 LMH for the TFC(PEG-b-PSF-b-PEG) and TFC(PSF) membranes. The XPS results confirmed that the annealing treatment did not change the crosslinking density of the polyamide through heating in water at $90{ }^{\circ} \mathrm{C}$ for $16 \mathrm{~h}$, as shown in Fig. 4b and Table S1. Moreover, the postannealing treatment dramatically decreased the $S$ of the TFC membrane with the block copolymer membrane support to $370 \pm 37 \mathrm{~nm}$, an $18 \%$ decrease compared with that of the TFC membrane without annealing. As a control, the annealing treatment did not change the $A, B$, or $S$ of the TFC FO membrane with the PSF support, as shown in Table S2 and Table S3. The post-annealing treatment did not change the structure or property of the polyamide in the TFC FO membranes (Figure S3) but did improve the support properties of the block copolymer membrane, with increased hydrophilicity and accelerated salt diffusion as discussed previously, benefiting from the directed self-assembly behavior of the polysulfone-based block copolymer membrane materials (Figure S4).

Therefore, it can be concluded that the post-annealing approach we developed can improve the water permeability of the TFC FO membrane having the polysulfone- 

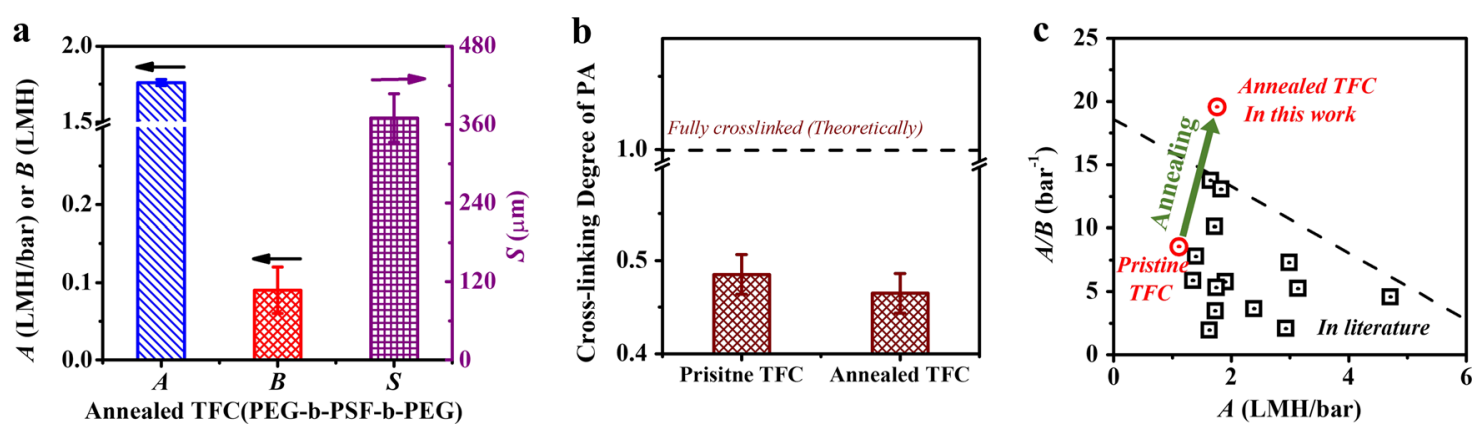

Fig. 4 The intrinsic transport parameters of annealed TFC(PEG-b-PSF-b-PEG) membranes, including the water permeability coefficient $A$, salt permeability coefficient $B$, and structural parameter $S(\mathbf{a})$, the crosslinking degree of the polyamide layer of the TFC(PEG-b-PSF-b-PEG) and annealed TFC(PEG-b-PSF-b-PEG) membranes (b), and the perm-selectivity ratio (ABB) vs the water permeability coefficient $A$ of the TFC FO membranes fabricated in this work and in the literature (c)

based block copolymer membrane support without sacrificing its selectivity. Compared with the reported properties of numerous TFC FO membranes, our TFC membrane with the block copolymer support presented a superior performance with the highest $A / B$ ratio of 19.6 $\mathrm{bar}^{-1}$ after the annealing treatment, significantly higher than the reported best value of $13.75 \mathrm{bar}^{-1}$ (Fig. 4c) ${ }^{14}$. Because of the high permeability and selectivity with a low structure parameter $S$, the annealed TFC FO membrane with the block copolymer membrane support achieved a very high water flux of $60 \mathrm{LMH}$ for the AL-DS orientation, very close to the reported highest value of $63 \mathrm{LMH}$ for the TFC polyamide membrane having an electrospun nanofibrous support under similar conditions using $2.0 \mathrm{M}$ $\mathrm{NaCl}$ as a draw solution ${ }^{8}$. The TFC FO membrane with the electrospun nanofibrous support generally had a very high reverse salt flux and a high $B$ value, along with low mechanical strength ${ }^{8,37,38}$, which brings difficulties to real applications.

Moreover, the water flux of the annealed TFC(PEG-bPSF-b-PEG) membrane increased significantly as the concentration of the draw solution $(\mathrm{NaCl})$ increased from 0.5 to $3.0 \mathrm{M}$ (Fig. 5a, b), and its flux increase rate was substantially higher than that of the TFC(PSF) control membrane. The water flux ratio $\left(J_{\mathrm{w}, \mathrm{AL}-\mathrm{FS}} / J_{\mathrm{w}, \mathrm{AL}-\mathrm{DS}}\right)$ for the two orientations of active layer facing the feed solution (AL-FS) and active layer facing the draw solution (AL-DS) is another indicator of ICP. A higher water flux ratio indicates a lower ICP due to a severe diffusive barrier in the tortuous polymeric support. Excitingly, the annealed TFC(PEG-bPSF-b-PEG) membrane possessed a $J_{\mathrm{w}, \mathrm{AL}-\mathrm{FS}} / J_{\mathrm{w}, \mathrm{AL}-\mathrm{DS}}$ in the range of $0.64-0.68$ for various draw concentrations, which was considerably higher than that of the reported TFC membrane in the range of $0.39-0.48^{14,39,40}$. This result suggests the ICP was significantly suppressed by the block copolymer support in comparison with the PSF support. Long-term stability tests (Fig. $5 \mathrm{c}, \mathrm{d}$ ) also showed that the annealed TFC(PEG-b-PSF-b-PEG) membrane had a very stable water flux profile with a suppressed flux decline compared with the control sample of TFC(PSF) when operating in the AL-DS mode with the same initial water flux. When using the same draw solution, the annealed TFC(PEG-b-PSF-b-PEG) membrane showed a significantly higher water flux, increased by $72 \%$ compared with the control TFC(PSF) membrane. Such high water flux and low flux decline with the annealed TFC(PEG-b-PSF-b-PEG) membrane resulted from the high permeability and selectivity of the membrane with reduced ICP.

As shown in Figure S6a, in the AL-FS mode, the reverse salt fluxes of the annealed TFC(PEG-b-PSF-b-PEG) and TFC(PSF) were very close when a low $\mathrm{NaCl}$ concentration solution $(0.5-2.0 \mathrm{M})$ was used as the draw solution. With increasing $\mathrm{NaCl}$ draw solution concentration from 2.5 to $3.0 \mathrm{M}$, the reverse salt flux value increased at a higher rate for the TFC(PSF) membrane than for the annealed TFC (PEG-b-PSF-b-PEG) membrane. This trend is more apparent when operated in the AL-DS mode (Figure S6b). These two different membranes presented similar low reverse salt fluxes under a relatively low $\mathrm{NaCl}$ draw concentration from 0.5 to $1.0 \mathrm{M}$, but the reverse salt flux of the TFC(PSF) membrane increased more rapidly than that of the annealed TFC(PEG-b-PSF-b-PEG) membrane as the $\mathrm{NaCl}$ concentration of the draw solution increased from 1.5 to $3.0 \mathrm{M}$.

Moreover, for a long-term test, the annealed TFC (PEG-b-PSF-b-PEG) membrane presented a slightly lower reverse salt flux than the control TFC(PSF) membrane (Figure S6c, d), under the operation conditions of maintaining the same initial flux or using the same $1 \mathrm{M} \mathrm{NaCl}$ draw solution. These results indicate that the annealed TFC(PEG-b-PSF-b-PEG) membrane performed significantly better in terms of both water permeability and salt selectivity than the control TFC (PSF) membrane. 

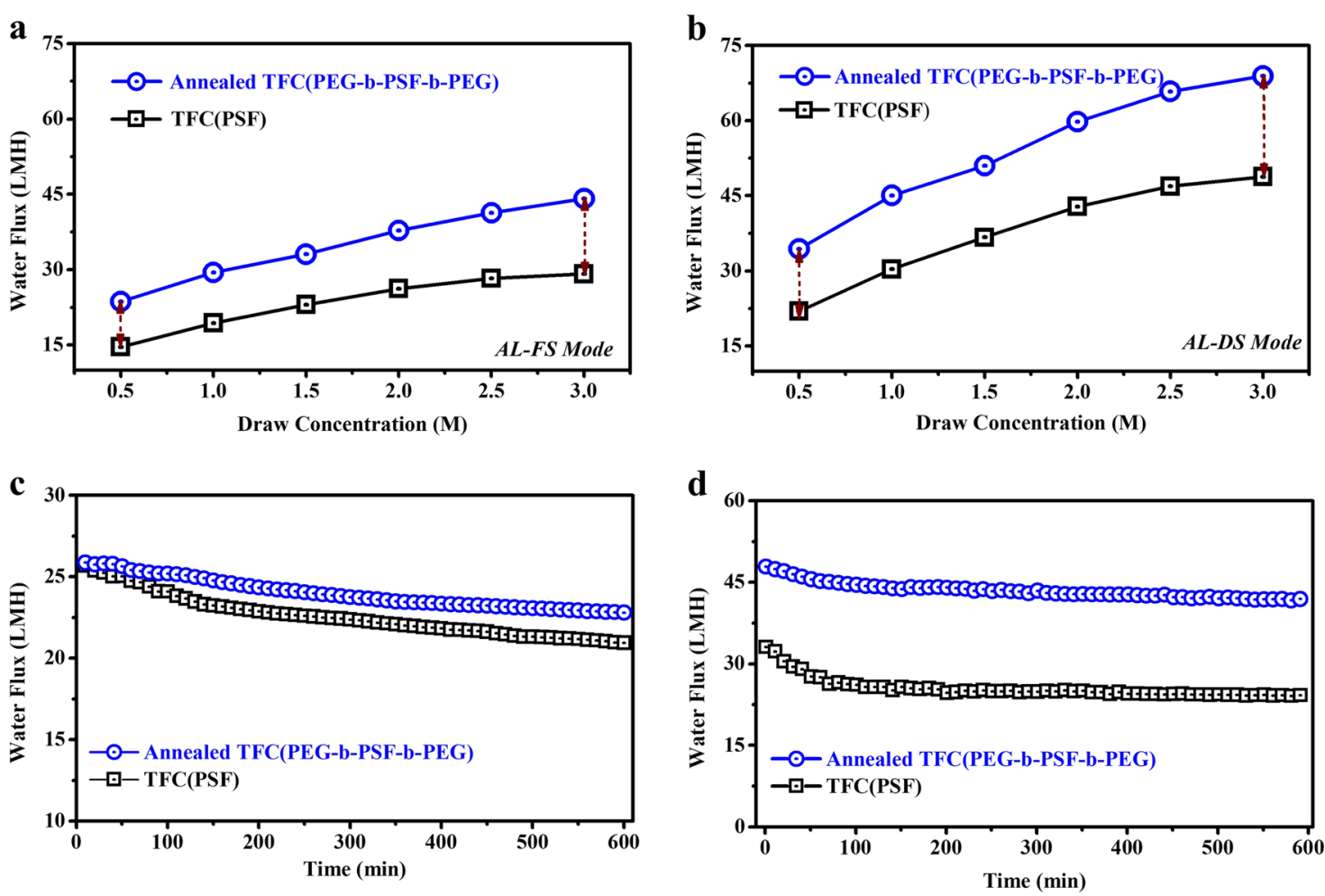

Fig. 5 Water flux of the TFC FO membranes under various concentrations of the NaCl draw solution operated in the AL-FS (a) and AL-DS (b) modes, and the long-term water flux of the annealed TFC(PEG-b-PSF-b-PEG) and TFC(PSF) membranes with the same initial water flux (c) and with the same $1 \mathrm{M} \mathrm{NaCl}$ draw solution (d) operated in the AL-DS mode

\section{The antifouling performance of the annealed TFC FO membrane having the block copolymer UF membrane support}

Because the post-annealing treatment improved the PEG surface enrichment and surface hydrophilicity of the polysulfone-based block copolymer membrane support of the TFC polyamide membrane (Figure S7), it may improve the membrane antifouling performance when the block copolymer membrane support faces the feed solution. Protein adsorption experiments were conducted to determine the antifouling performance of the block copolymer membrane support of the annealed TFC polyamide membrane. FITC-labeled BSA was used as the model protein foulant ${ }^{41}$. After a $2 \mathrm{~h}$ incubation of the FITC-labeled BSA and the block copolymer membrane support of the annealed TFC polyamide membrane and 3 times thorough cleaning, confocal laser scanning microscopy was used to observe the residual BSA absorbed on the membrane surface. Figure $6 \mathrm{a}, \mathrm{b}$ shows that a significantly reduced green fluorescence from the FITC-labeled BSA was observed on the support of the annealed TFC(PEG-b-PSF-b-PEG) membrane compared with that on the control TFC(PSF) membrane, indicating that the annealed block copolymer membrane support had improved antifouling properties by preventing the adsorption of BSA. Furthermore, dynamic protein fouling experiments were performed in the crossflow FO unit. As shown in Fig. 6c, for the annealed TFC (PEG-b-PSF-b-PEG) membrane, after $24 \mathrm{~h}$ of operation, the percentage of membrane water flux decline due to protein fouling was $39.1 \%$ of the initial water flux, significantly lower than the value of $52.4 \%$ for the control TFC(PSF) membrane. Osmotic backwash was then performed to clean the BSA-fouled membrane and recover the water flux. The results reveal that an $80.6 \%$ water flux recovery was achieved for the annealed TFC(PEG-b-PSF-b-PEG) membrane, substantially higher than the value of $63.9 \%$ for the control TFC(PSF) membrane. Quantitative analysis of the absorbed protein on the support surface of the TFC polyamide membrane revealed that $283 \pm 80$ and $83 \pm$ $14 \mu \mathrm{g} / \mathrm{cm}^{2}$ of the total protein were accumulated on the annealed TFC(PEG-b-PSF-b-PEG) membrane during the fouling and cleaning processes, significantly lower than the values of $713 \pm 155$ and $375 \pm 81 \mu \mathrm{g} / \mathrm{cm}^{2}$ for the control TFC(PSF) membrane (Fig. 6d). Altogether, these results indicate that the decreased protein absorption on the support surface is mainly responsible for the reduced water flux decline of the annealed TFC(PEG-b-PSF-b-PEG) membrane under dynamic protein fouling conditions. 


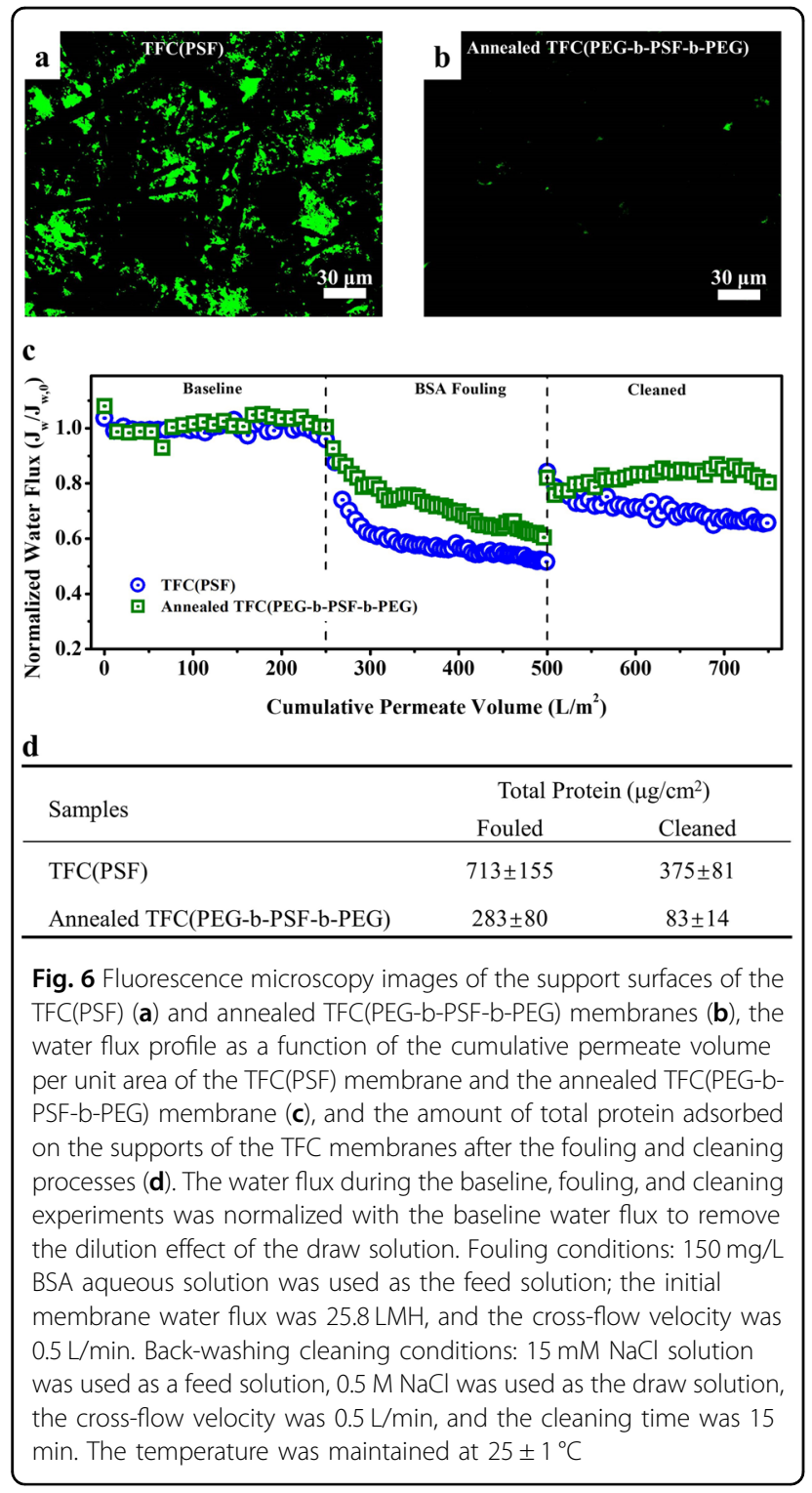

\section{Conclusions}

In this work, we used an amphiphilic polysulfone-based block copolymer (PEG-b-PSF-b-PEG) membrane as the support to fabricate a high-performance TFC FO membrane and developed a facile post-annealing approach to simultaneously improve the water permeability and antifouling property of the TFC FO membrane without sacrificing its selectivity. Our results show that the pristine block copolymer membrane support provided a favorable surface to form a highly crosslinked polyamide with a very low reverse salt flux. The post-annealing treatment tailored the membrane structure and surface properties of the block copolymer membrane support to improve the water flux of the fabricated TFC FO membrane and to decrease its structure parameter $S$ by taking advantage of the self-assembly behavior of the block copolymer
PEG-b-PSF-b-PEG. The annealed TFC FO membrane with the block copolymer membrane support exhibited a superior performance with the highest $A / B$ ratio of 19.6 $\mathrm{bar}^{-1}$, substantially higher than the reported high value of $13.75 \mathrm{bar}^{-1}$. Excitingly, the membrane also achieved a very high water flux of $60 \mathrm{LMH}$ and a very low reverse salt flux of $4 \mathrm{gMH}$ in the AL-DS mode of the FO process. Moreover, the membrane presented a high $J_{\mathrm{w}, \mathrm{AL}-\mathrm{FS}} / J_{\mathrm{w}, \mathrm{AL}-\mathrm{DS}}$ in the range of $0.64-0.68$ for various draw solution concentrations, considerably higher than that of the reported TFC FO membrane in the range of 0.39-0.48, suggesting suppressed ICP. Our research therefore develops the distinctive block copolymer membrane material PEG-b-PSF-b-PEG to fabricate the TFC membrane support, provides experimental evidence to identify the impact of the support on the formation of polyamide, and demonstrates a facile postannealing treatment to break the permeability-selectivity trade-off of the TFC FO membrane and enhance its antifouling properties. It is worth mentioning that the PEG-bPSF-b-PEG block copolymer can also be used to cast membrane supports for the fabrication of TFC polyamide membranes working as a reverse osmosis (RO) membrane and a nanofiltration (NF) membrane.

\section{Acknowledgements}

The authors gratefully thank the funding support from the National Natural Science Foundation of China (Nos. 21476249 and 51708408), Program for Innovative Research Team in University of Tianjin (No. TD13-5044), Chang-jiang Scholars and Innovative Research Team in the University of Ministry of Education, China (No. IRT-17R80), and the Science and Technology Plans of Tianjin (No. 17PTSYJC00060).

\section{Author details}

${ }^{1}$ State Key Laboratory of Separation Membranes and Membrane Processes, National Center for International Research on Membrane Science and Technology, School of Materials Science and Engineering, Tianjin Polytechnic University, Tianjin 300387, PR China. ${ }^{2}$ CAS Key Laboratory of Coastal Environmental Processes and Ecological Remediation, Research Center for Coastal Environmental Engineering and Technology of Shandong Province, Yantai Institute of Coastal Zone Research, Chinese Academy of Sciences, Yantai, Shandong Province 264003, PR China. ${ }^{3}$ University of Chinese Academy of Sciences, Beijing 100049, PR China

Conflict of interest

The authors declare that they have no conflict of interest.

\section{Publisher's note}

Springer Nature remains neutral with regard to jurisdictional claims in published maps and institutional affiliations.

Supplementary information is available for this paper at https://doi.org/ 10.1038/s41427-019-0114-1.

Received: 30 October 2018 Revised: 24 December 2018 Accepted: 13 January 2019.

Published online: 21 March 2019

\footnotetext{
References

1. Logan, B. E. \& Elimelech, M. Membrane-based processes for sustainable power generation using water. Nature 488, 313-319 (2012).
} 
2. Elimelech, M. \& Phillip, W. A. The future of seawater desalination: energy, technology, and the environment. Science 333, 712-717 (2011).

3. Coday, B. D. et al. The sweet spot of forward osmosis: treatment of produced water, drilling wastewater, and other complex and difficult liquid streams. Desalination 333, 23-35 (2014).

4. Han, G., Chan, S. S. \& Chung, T.-S. Forward osmosis (FO) for water reclamation from emulsified oil/water solutions: effects of membrane and emulsion characteristics. ACS Sustain. Chem. Eng. 4, 5021-5032 (2016).

5. Yip, N. Y., Tiraferri, A., Phillip, W. A., Schiffman, J. D. \& Elimelech, M. High performance thin-film composite forward osmosis membrane. Environ. Sci. Technol. 44, 3812-3818 (2010).

6. Shannon, M. A. et al. Science and technology for water purification in the coming decades. Nature 452, 301-310 (2008).

7. Hoover, L. A., Phillip, W. A., Tiraferri, A., Yip, N. Y. \& Elimelech, M. Forward with osmosis: emerging applications for greater sustainability. Environ. Sci. Technol. 45, 9824-9830 (2011)

8. Song, X., Liu, Z. \& Sun, D. D. Nano gives the answer: breaking the bottleneck of internal concentration polarization with a nanofiber composite forward osmosis membrane for a high water production rate. Adv. Mater. 23, 3256-3260 (2011).

9. Shaffer, D. L., Werber, J. R., Jaramillo, H., Lin, S. \& Elimelech, M. Forward osmosis: where are we now? Desalination 356, 271-284 (2015).

10. Akther, $\mathrm{N}$. et al. Recent advancements in forward osmosis desalination: a review. Chem. Eng. J. 281, 502-522 (2015)

11. Tiraferri, A., Yip, N. Y., Phillip, W. A., Schiffman, J. D. \& Elimelech, M. Relating performance of thin-film composite forward osmosis membranes to support layer formation and structure. J. Membr. Sci. 367, 340-352 (2011).

12. Obaid, $\mathrm{M}$. et al. Amorphous $\mathrm{SiO}_{2} \mathrm{NP}$-incorporated poly(vinylidene fluoride) electrospun nanofiber membrane for high flux forward osmosis desalination. ACS Appl. Mater. Interfaces 8, 4561-4574 (2016).

13. Duong, P. H. et al. Hydroxyl functionalized polytriazole-co-polyoxadiazole as substrates for forward osmosis membranes. ACS Appl. Mater. Interfaces 7 3960-3973 (2015).

14. Xiao, P. et al. A sacrificial-layer approach to fabricate polysulfone support for forward osmosis thin-film composite membranes with reduced internal concentration polarisation. J. Membr. Sci. 481, 106-114 (2015).

15. Werber, J. R., Osuji, C. O. \& Elimelech, M. Materials for next-generation desalination and water purification membranes. Nat. Rev. Mater. 1, 16018 (2016).

16. Ghosh, A. K. \& Hoek, E. M. V. Impacts of support membrane structure and chemistry on polyamide-polysulfone interfacial composite membranes. J. Membr. Sci. 336, 140-148 (2009).

17. Wei, J., Qiu, C., Tang, C. Y., Wang, R. \& Fane, A. G. Synthesis and characterization of flat-sheet thin film composite forward osmosis membranes. J. Membr. Sci. 372, 292-302 (2011)

18. Raaijmakers, M. J. T. \& Benes, N. E. Current trends in interfacial polymerization chemistry. Prog. Polym. Sci. 63, 86-142 (2016).

19. Wang, N., Wang, T. \& Hu, Y. Tailoring membrane surface properties and ultrafiltration performances via the self-assembly of polyethylene glycol-blockpolysulfone-block-polyethylene glycol block copolymer upon thermal and solvent annealing. ACS Appl. Mater. Interfaces 9, 31018-31030 (2017).

20. Lu, X., Arias Chavez, L. H., Romero-Vargas Castrillon, S., Ma, J. \& Elimelech, M. Influence of active layer and support layer surface structures on organic fouling propensity of thin-film composite forward osmosis membranes. Environ. Sci. Technol. 49, 1436-1444 (2015).

21. Yin, J. et al. Membranes with highly ordered straight nanopores by selective swelling of fast perpendicularly aligned block copolymers. ACS Nano 7, 9961-9974 (2013).

22. Huang, L. \& McCutcheon, J. R. Impact of support layer pore size on performance of thin film composite membranes for forward osmosis. J. Membr. Sci. 483, 25-33 (2015).
23. Liu, Z. \& Hu, Y. Sustainable antibiofouling properties of thin film composite forward osmosis membrane with rechargeable silver nanoparticles loading. ACS Appl. Mater. Interfaces 8, 21666-21673 (2016).

24. Tiraferri, A., Yip, N. Y., Straub, A. P., Romero-Vargas Castrillon, S. \& Elimelech, M A method for the simultaneous determination of transport and structural parameters of forward osmosis membranes. J. Membr. Sci. 444, 523-538 (2013).

25. Furuike, T., Chaochai, T., Komoto, D. \& Tamura, H. Adsorption and desorption behaviors of bovine serum albumin on gelatin/chitosan sponge. J. Mater. Sci. Chem. Eng. 05, 109-120 (2017).

26. Yip, N. Y. \& Elimelech, M. Influence of natural organic matter fouling and osmotic backwash on pressure retarded osmosis energy production from natural salinity gradients. Environ. Sci. Technol. 47, 12607-12616 (2013).

27. Zhao, J. et al. Improved biocompatibility and antifouling property of polypropylene non-woven fabric membrane by surface grafting zwitterionic polymer. J. Membr. Sci. 369, 5-12 (2011).

28. Oh, S. J., Kim, N. \& Lee, Y. T. Preparation and characterization of PVDF/TiO organic-inorganic composite membranes for fouling resistance improvement. J. Membr. Sci. 345, 13-20 (2009).

29. Behboudi, A., Jafarzadeh, Y. \& Yegani, R. Enhancement of antifouling and antibacterial properties of PVC hollow fiber ultrafiltration membranes using pristine and modified silver nanoparticles. J. Environ. Chem. Eng. 6, 1764-1773 (2018).

30. Yang, Y., Zhang, H., Wang, P., Zheng, Q. \& Li, J. The influence of nano-sized $\mathrm{TiO}_{2}$ fillers on the morphologies and properties of PSF UF membrane. J. Membr. Sci. 288, 231-238 (2007).

31. Fan, Z., Wang, Z., Sun, N., Wang, J. \& Wang, S. Performance improvement of polysulfone ultrafiltration membrane by blending with polyaniline nanofibers. J. Membr. Sci. 320, 363-371 (2008).

32. Romero-Vargas Castrillón, S., Lu, X., Shaffer, D. L. \& Elimelech, M. Amine enrichment and poly(ethylene glycol) (PEG) surface modification of thin-film composite forward osmosis membranes for organic fouling control. J. Membr. Sci. 450, 331-339 (2014).

33. Peinemann, K. V., Abetz, V. \& Simon, P. F. Asymmetric superstructure formed in a block copolymer via phase separation. Nat. Mater. 6, 992-996 (2007).

34. Phillip, W. A. et al. Tuning structure and properties of graded triblock terpolymer-based mesoporous and hybrid films. Nano Lett. 11, 2892-2900 (2011).

35. Wang, J.-J., Yang, H.-C., Wu, M.-B., Zhang, X. \& Xu, Z.-K. Nanofiltration membranes with cellulose nanocrystals as an interlayer for unprecedented performance. J. Mater. Chem. A 5, 16289-16295 (2017).

36. Tian, M., Qiu, C., Liao, Y., Chou, S. \& Wang, R. Preparation of polyamide thin film composite forward osmosis membranes using electrospun polyvinylidene fluoride (PVDF) nanofibers as substrates. Sep. Purif. Technol. 118, 727-736 (2013).

37. Bui, N. N. \& McCutcheon, J. R. Hydrophilic nanofibers as new supports for thin film composite membranes for engineered osmosis. Environ. Sci. Technol. 47 1761-1769 (2013)

38. Puguan, J. M. C., Kim, H.-S., Lee, K.-J. \& Kim, H. Low internal concentration polarization in forward osmosis membranes with hydrophilic crosslinked PVA nanofibers as porous support layer. Desalination 336, 24-31 (2014).

39. Liang, H.Q. et al. Forward osmosis membranes with unprecedented water flux. J. Membr. Sci. 529, 47-54 (2017).

40. Li, X.-M. et al. Origin of delamination/adhesion in polyetherimide/polysulfone co-cast membranes. J. Membr. Sci. 352, 173-179 (2010).

41. Liu, Z. Y. et al. Modification of thin film composite polyamide membranes with 3D hyperbranched polyglycerol for simultaneous improvement in their filtration performance and antifouling properties. J. Mater. Chem. A 5, 23190-23197 (2017). 